\title{
A STUDY OF THE EFFECT OF ELECTRO-SLAG RE-MELTING PARAMETERS ON THE STRUCTURAL INTEGRITY OF LARGE DIAMETER ALLOY 718 ESR INGOT
}

\author{
D. G. Evans ${ }^{1}$ and M. Fahrmann ${ }^{2}$ \\ ${ }^{1}$ Special Metals Corporation, New Hartford, New York \\ ${ }^{2}$ Special Metals Corporation, Huntington, West Virginia
}

Keywords : wrought Ni-base superalloy, electro-slag remelting, melt pool dynamics, niobium, macrosegregation, microsegregation

\begin{abstract}
$\underline{\text { Abstract }}$
The effect of electro-slag re-melting (ESR) parameters on the structural integrity of nominally (33-35) inch $(838-890 \mathrm{~mm})$ diameter ingot in alloy 718 was studied. Chiefly, the melt rate was varied between $50 \%$ and $130 \%$ relative to a baseline. The corresponding melt pool profiles were tracked, and associated macro- and micro-segregation patterns were investigated. Their implications for the structural soundness of the ingot during subsequent processing are discussed.
\end{abstract}

\section{Introduction}

The drive for increased operating efficiency of utility gas turbines has resulted in the need for larger, yet structurally sound and chemically homogeneous ingots for forging stock in Nibase disk superalloys such as alloy 718 [1].

One important process step in the production of these large ingots is electro-slag re-melting (ESR) of primary feedstock into feedstock for the final melting operation, vacuum arc re- melting (VAR). The structural soundness of this intermediate ESR ingot directly affects its resistance against cracking due to thermal stress in the course of VAR itself, or due to mechanical stress during any intermediate forging operations prior to VAR. It is worth noting that these large-diameter ingots, which weigh roughly 14 tons $(30,000 \mathrm{lbs}$.), would typically result in only one or two finished parts. Thus, any re-melt anomalies during final VAR caused by poor electrode quality will result in major yield losses.

\section{Experiment}

A systematic study of ESR melting parameters, chiefly melt rate, was undertaken for ingot sizes ranging in diameter from 33" to 35 ". Standard start-up and extended hot topping practices were employed.

The initial vacuum induction-melted (VIM) electrode was in all instances nominally 31 inch $(787 \mathrm{~mm})$ in diameter, melted to a com- 
positional aim typical of alloy $718^{1}$ for large power generation gas turbine rotors. Most prominently, this concerned a niobium content of about $5.0 \%$ by mass, the latter being markedly below aerospace grade requirements, and a carbon content of $0.01 \%$.

The study consisted of experiments at two different SMC facilities. The first set, executed at the New Hartford (NH) facility, involved the manufacture of a 33 inch $(838 \mathrm{~mm})$ diameter by 132 inch $(3353 \mathrm{~mm})$ long ESR ingot melted at four different melt main rates. The four melt rates were selected to straddle a common melt rate for this ingot size and application. The aim melt rate being referred to as $100 \%$, the other three melt rates were 63 $\%, 77 \%$, and $130 \%$ relative to that aim. The melt program transitioned the melt rates between each step over roughly 1,000 lbs. (460 $\mathrm{kg}$ ) of melting. The furnaces employed use a PI-based current control to set and hold the desired melt rate.

The progression of the melt pool was marked at certain intervals by simultaneously emptying two $750-\mathrm{ml}$ beakers filled with nickel balls into the melt. The beakers were positioned 180 degrees apart on either side of the crucible. The nickel balls disrupt the local solidification front and, thus, delineate the pool contour. Once the melt was completed, the entire ingot was sectioned longitudinally. This allowed the local ingot structure to be correlated with then-existent melt parameters. The slices were conditioned, and sequentially etched with both a mixture of hydrochloric acid / hydrogen peroxide and Canada's etch to reveal the mac-

\footnotetext{
${ }^{1}$ A typical composition (in mass $\%$ ) is $0.01 \mathrm{C}$, 18.8Fe, 18.0Cr, 3.0Mo, 0.5Al, 0.9Ti, 5.0Nb, bal. Ni
}

rostructure and any segregation patterns, respectively.

Based on the outcome of the New Hartford experiment, an even lower main step melt rate of $50 \%$ was selected for two full-scale $35 \%$ $(890 \mathrm{~mm})$ trials at the Huntington (HU) facility. In addition, a third VIM electrode was remelted at the $77 \%$ melt rate in order to verify reproducibility of some of the findings at the New Hartford facility. Remarkably, the ingot surface quality was acceptable for even the lowest melt rate.

All Huntington ESR ingots were subsequently forged back to nominally 27 inch $(686 \mathrm{~mm})$ diameter rods after having received special post-ESR heat treatments. Head (top) and toe (bottom) crops were taken at this stage, from which longitudinal slices were obtained for the examination of macro- and microstructure.

The two forged-back ingots re-melted at the low rate of $50 \%$ required only standard crops to reach sound material. In contrast, the ingot re-melted at the $77 \%$ melt rate exhibited massive centerline bursts as verified by ultrasonic testing. These bursts extended from the ingot head all the way back to within roughly one diameter up from the ingot toe.

\section{$\underline{\text { Results }}$}

The actual melt rate data is shown in Fig. 1. The melting at the lower melt rates went smoothly. At the higher melt rates, there were indications of electrode cracking manifesting themselves in a sharp rise and drops in the melt rate. 


\section{$\underline{\text { Fig. } 1}$ : Melt rate and Ni pool marking locations}

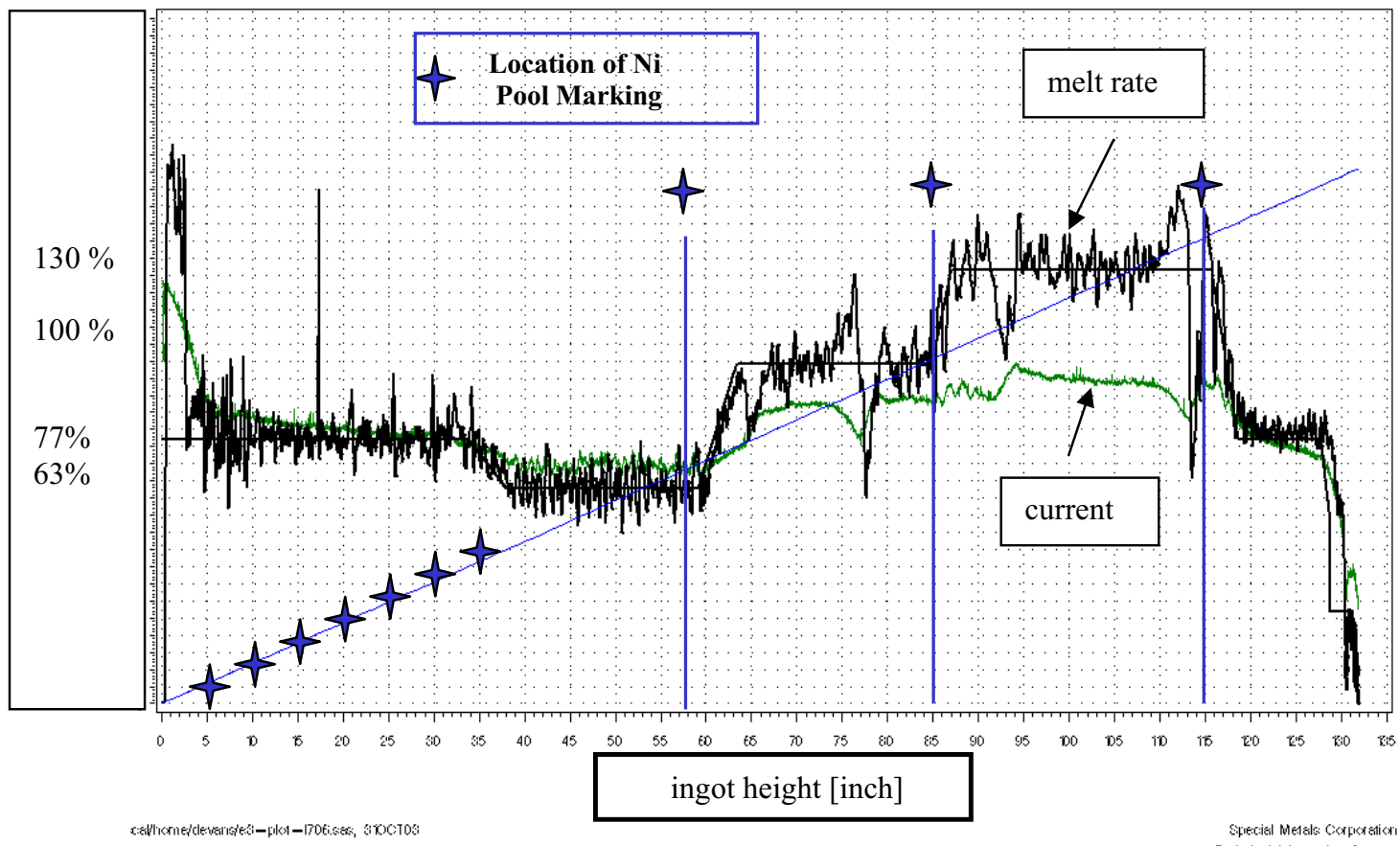

Fig. 2 displays macro-etched longitudinal slices of the very ingot taken at completion of the respective melt rate segment. Shown in several instances are also adjacent ingot sections etched with Canada's reagent to highlight any macro-segregation. The slice pertaining to the baseline melt rate is not shown for reasons stated farther below.

Fig. 2 : Macro-photos of longitudinal etch plates obtained from the various main melt rate segments. The number labels signify the respective ingot height. Prominent macrosegregation patterns are indicated by arrows.

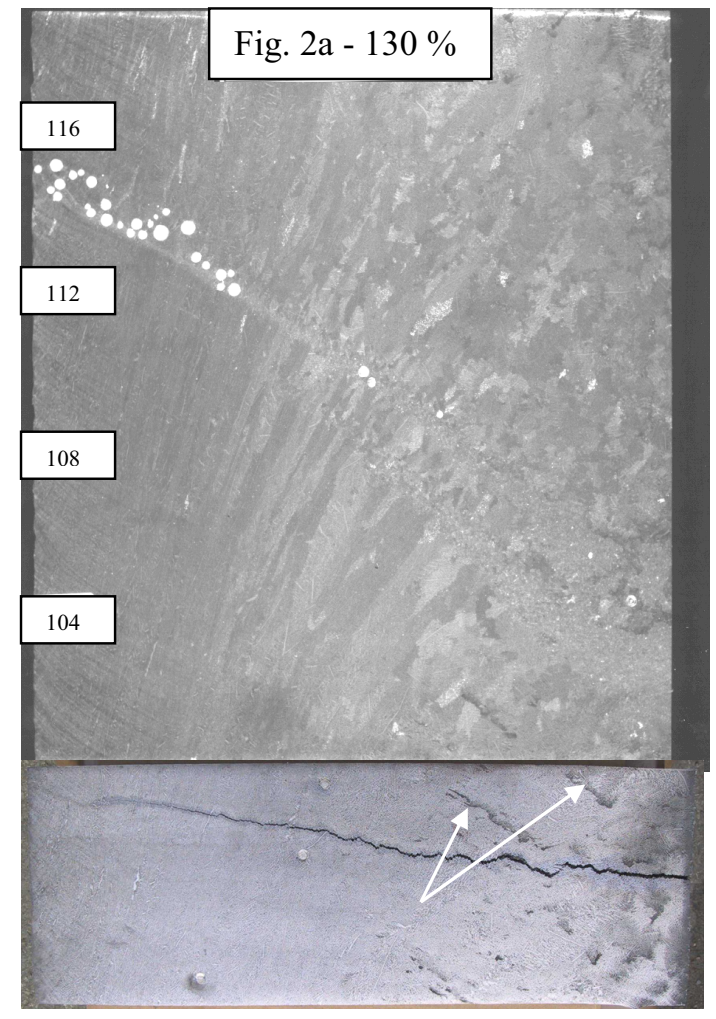




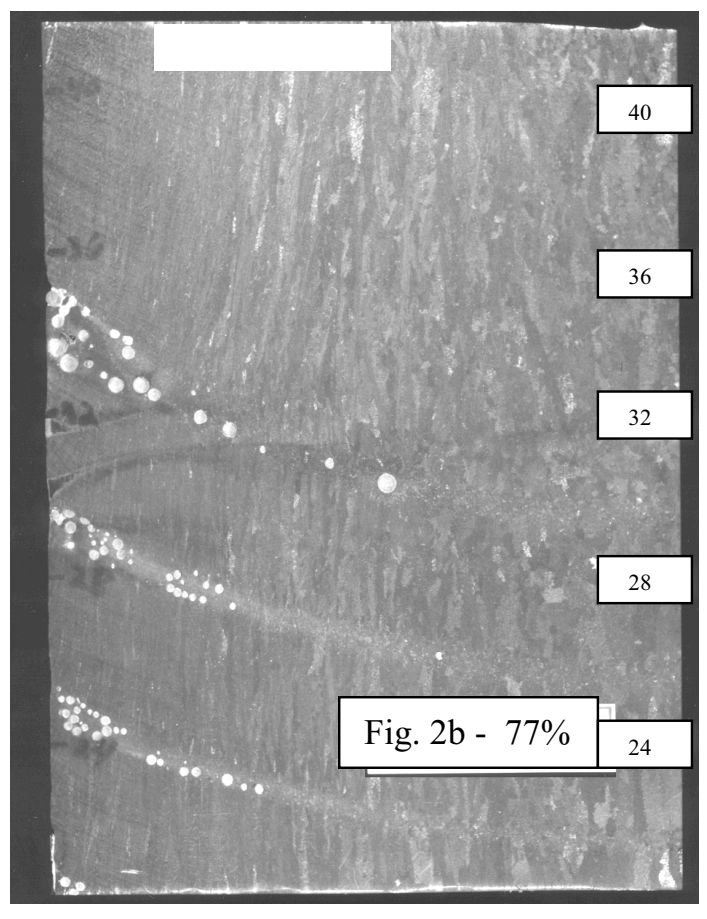

The macro-etch results demonstrate that none of the melt rates in the chosen range produced a macro-segregation-free product. The appearance of the macro-segregation changed from vertical freckle columns at and near the centerline to freckle bands that followed the pool contour as the melt rate increased.

The major crack discernable in the bottom slice of the $130 \%$ melt rate samples is indicative of the poor fracture toughness of the material at this stage.

Macro-photographs of the billet head slices that resulted from the full-scale $77 \%$ and 50 $\%$ melt rate trials in Huntington are shown in Fig. 3.

Fig. 3 : Macro-photographs of sections of the heads (tops) of the forged-back, full-scale Huntington alloy 718 trial pieces.
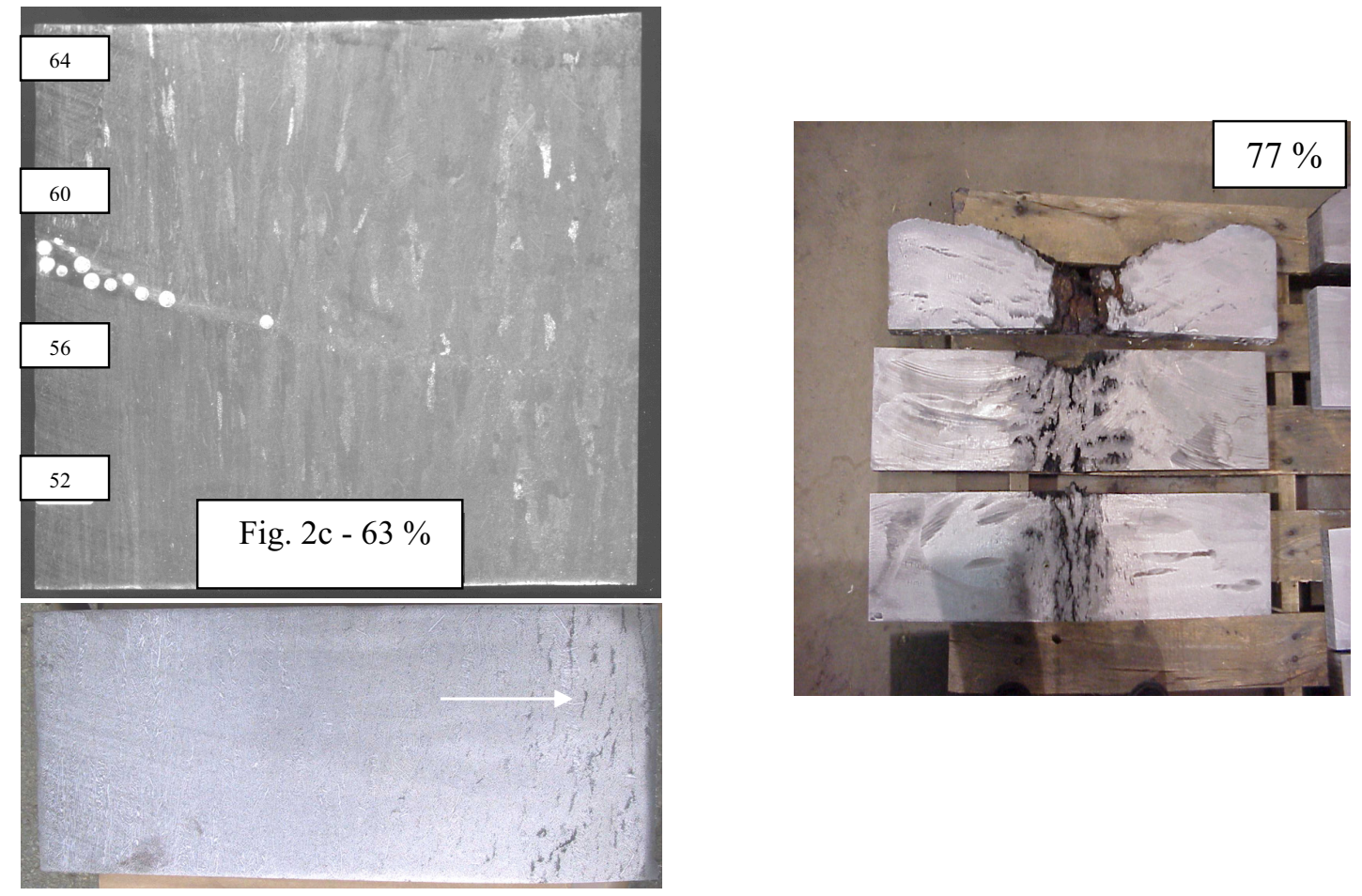


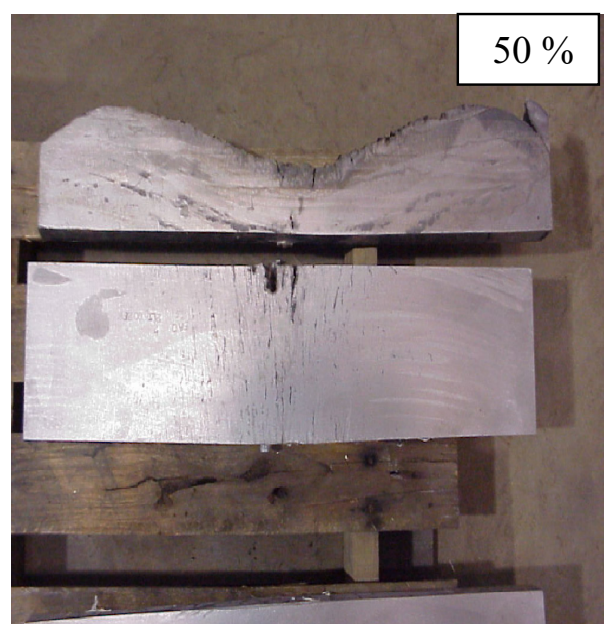

The $77 \%$ melt rate material exhibited massive vertical freckle channels similar to those experienced in the New Hartford trial at nominally the same melt rate. These massive channels presumably caused the ingot to crack during forge-back. Notice that the vertical freckle channels are present even at the lowest melt rate of $50 \%$, however, to a much lesser extent. These rather isolated freckles were in many instances associated with micro-cracks. None of the toe (bottom) billet slices exhibited any freckles.

The pool profiles as delineated by the nickel balls in Figs. 2 displayed the expected transition from a shallow bowl-shaped pool (for relatively low melt rates) to the classical "V"shaped pool (at high melt rates). The pool pertaining to the baseline $100 \%$ melt rate was rather unusual in that it was very deep and " $U$ " shaped. Similar abnormally deep ESR pools have been observed in the past for little understood reasons.
The measured pool depths as a function of melt rate are presented in Fig. 4.

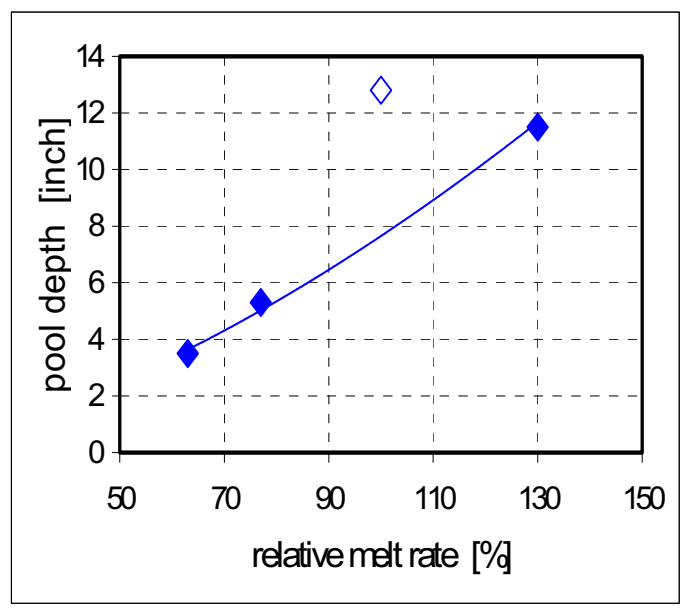

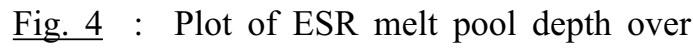
relative melt rate. A power fit is superimposed on the data points.

The height of the liquid metal head (the distance between the pool surface and the first frozen metal on the side adjacent to the crucible wall) was neglected in these measurements.

In addition to the pool depth measurements, the chemical composition was probed at center, mid-radius, and edge locations roughly following the local pool contour. Both the $\mathrm{x}$ ray fluorescence and ICP method were employed for the analyses. The respective areas sampled were of the order of $10 \times 10 \mathrm{~mm}^{2}$ and greater, and, thus, contained numerous solidification dendrites.

Hence, the chemical compositions quoted in Table 1 may be considered bulk compositions. Only elements that exhibited a significant variation were included in this table. 
Table 1 : Selected chemical composition at specified center (C), mid-radius (M), and edge (E) locations in the alloy 718 ingot / forgebacks. The actual / equivalent axial location measured from the respective ingot bottom is indicated by $h$.

\begin{tabular}{|c|c|c|c|c|c|}
\hline MR & $\mathbf{h}$ & $\mathbf{C}$ & $\mathbf{N b}$ & $\mathbf{F e}$ & $\mathbf{C r}$ \\
\hline$[\%]$ & [inch] & {$[\mathrm{wt} \%]$} & {$[\mathrm{wt} \%]$} & {$[\mathrm{wt} \%]$} & {$[\mathrm{wt} \%]$} \\
\hline \multicolumn{6}{|c|}{ measurements on as-cast $\mathrm{NH}$ ingot } \\
\hline 63 & $60 \mathrm{C}$ & 0.012 & 5.14 & 17.8 & 18.0 \\
\hline “ & “M & 0.010 & 4.96 & 18.0 & 18.1 \\
\hline "، & " $\mathrm{E}$ & 0.015 & 4.96 & 17.9 & 18.1 \\
\hline 77 & $8 \mathrm{C}$ & 0.013 & 4.97 & 18.2 & 18.0 \\
\hline "“ & "M & 0.013 & 4.98 & 18.2 & 18.0 \\
\hline "“ & "E & 0.014 & 4.94 & 18.3 & 18.0 \\
\hline 77 & $36 \mathrm{C}$ & 0.013 & 5.41 & 17.5 & 17.8 \\
\hline " & "M & 0.015 & 4.92 & 17.9 & 18.1 \\
\hline " & "E & 0.013 & 4.97 & 17.9 & 18.0 \\
\hline 77 & $124 \mathrm{C}$ & 0.024 & 6.25 & 17.8 & 17.1 \\
\hline "، & " $\mathrm{M}$ & 0.008 & 4.95 & 18.5 & 18.0 \\
\hline "، & "E & 0.009 & 5.24 & 18.3 & 17.8 \\
\hline 130 & $115 \mathrm{C}$ & 0.012 & 5.00 & 18.0 & 18.1 \\
\hline "، & " $\mathrm{M}$ & 0.015 & 5.04 & 17.9 & 18.1 \\
\hline "، & "E & 0.013 & 5.05 & 17.8 & 18.1 \\
\hline \multicolumn{6}{|c|}{ measurements on HU forge-back I } \\
\hline 50 & $109 \mathrm{C}$ & 0.009 & 5.20 & 18.3 & 18.1 \\
\hline "6 & " $\mathrm{M}$ & 0.009 & 4.93 & 18.5 & 18.2 \\
\hline 66 & " $\mathrm{E}$ & 0.009 & 4.88 & 18.5 & 18.2 \\
\hline \multicolumn{6}{|c|}{ measurements on HU forge-back II } \\
\hline 50 & $110 \mathrm{C}$ & 0.009 & 5.24 & 18.6 & 17.9 \\
\hline "6 & "M & 0.009 & 5.13 & 18.7 & 18.0 \\
\hline 6 & "E & 0.009 & 5.03 & 18.7 & 18.0 \\
\hline \multicolumn{6}{|c|}{ measurements on HU forge-back III } \\
\hline 77 & $72 \mathrm{C}$ & 0.010 & 5.44 & 17.9 & 17.9 \\
\hline 6 & "M & 0.007 & 4.90 & 18.4 & 18.1 \\
\hline " & " $E$ & 0.011 & 4.96 & 18.4 & 18.1 \\
\hline "6 & $106 \mathrm{C}$ & 0.024 & 5.73 & 17.9 & 17.9 \\
\hline "6 & "M & 0.008 & 4.95 & 18.4 & 18.1 \\
\hline " & "E & 0.010 & 4.92 & 18.2 & 18.2 \\
\hline
\end{tabular}

For some combinations of melt rate and ingot height, the ESR ingot center was found to be significantly enriched in niobium. These combinations are highlighted in Table 1. The corresponding differential in $\mathrm{Nb}$ between center and edge is plotted in Fig. 5.

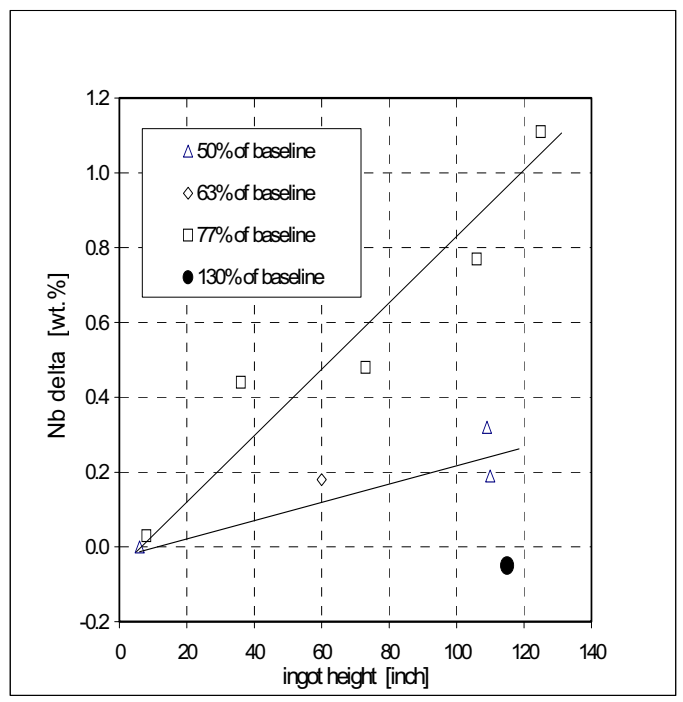

Fig. 5 : The differential in $\mathrm{Nb}$ between center and edge is plotted as a function of ingot height and melt rate. The straight lines are drawn as a guide for the eye only.

The results obtained from the independent $\mathrm{NH}$ and HU $77 \%$ melt rate trials match remarkably well. The result from the NH 130\% melt rate segment was initially questioned, however, repetitive measurements on a total of six centerline or near-centerline samples confirmed the original findings.

Examination of the microstructure corresponding to the freckled regions depicted in bottom Fig. 3 revealed the presence of clusters of large, blocky precipitates that were found to be rich in niobium and molybdenum, and pre- 
sumed to be Laves phase (Fig. 6). These clusters were in many cases micro-cracked.

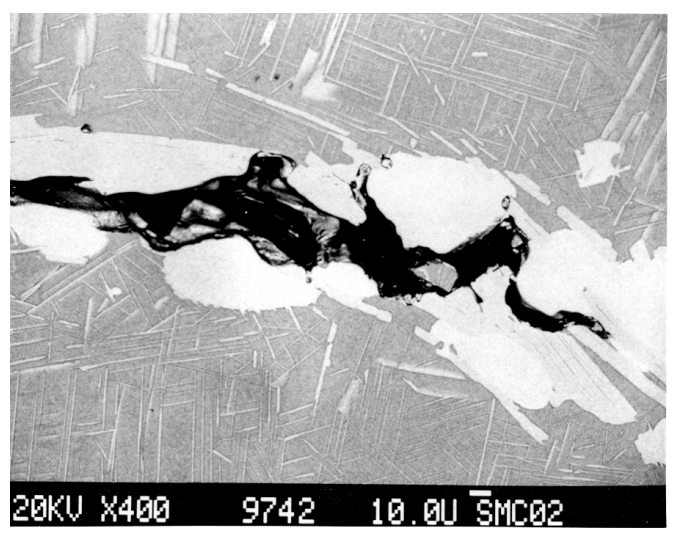

Fig. 6 : Close-up of a freckle and associated micro-cracks in forged-back alloy 718 ESR ingot, re-melted at the lowest melt rate of 50 $\%$. A blocky phase (presumed to be Laves), and an acicular phase (presumed to be delta phase) are discernable. Back-scattered electron image.

It is noted that the bulk of the delta needles / plates formed during slow cool-down from the forge-back temperature.

The examination of the heavy freckle channels apparent in the top Fig. 3 was compounded by oxidation. The second phase(s) observed on either crack surface looked heavily disintegrated, and were found to be rich in $\mathrm{Nb}, \mathrm{Mo}$, $\mathrm{Cr}$, and $\mathrm{Ti}$ relative to the matrix. A mix of Laves phase, delta, and primary carbides (recalling the local carbon content of $0.024 \%$ ) seems likely in these channels in accordance with literature [2].

Clusters of similar appearance were also observed to exist in the material after reannealing at the forging temperature, and even after a post-forge standard 2-step homogenization treatment.

Simulating the scenario of one of the heaviest cases of enrichment in $\mathrm{Nb}$ measured by means of the CALPHAD software Thermo-Calc [3] suggested the presence of essentially singlephase austenite only (the dot in Fig. 7) in thermodynamic equilibrium at $1182{ }^{\circ} \mathrm{C}$ $\left(2150^{\circ} \mathrm{F}\right)$.

Hence, sufficient exposure to this temperature should dissolve all Laves / delta. This, however, was not found to be the case even after extended laboratory heat treatments (288 hours) beyond any practical limits. This indicates that ESR alloy 718 ingots are likely to contain residual Laves phase during all subsequent stages of processing.

\section{DISCUSSION}

All of the above findings consistently point at the freckle formation propensity of alloy 718 during ESR, be it in smaller diameters for aerospace-grade [4], or comparatively larger diameters for grades reduced in niobium as employed in this study. The very sluggishness of reaching thermodynamic equilibrium, once macro-segregation of niobium is present, is also apparent.

Nevertheless, a process window was identified that sufficiently suppressed the occurrence of freckles, thus allowing an acceptable feedstock for the final melt conversion step to be made. 
THERMO-CALC ( $04.02 .17: 12.03):$

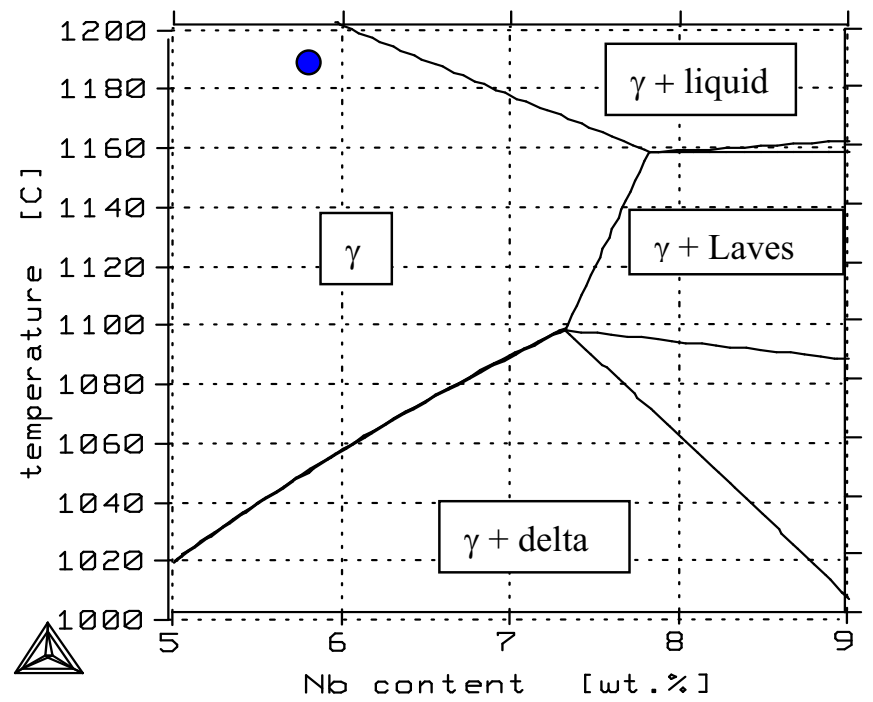

Fig. 7 : Thermo-Calc simulation of the phase fields at as a function of temperature and bulk niobium content.

Indeed, it is noted that the surface quality of the two low-melt rate (50\%) ESR ingots was markedly better than anticipated, and that both pieces resulted in stable VAR traces. This, in turn, produced chemically homogeneous and structurally sound 30 inch $(760 \mathrm{~mm})$ diameter triple-melted ingots.

The above emphasis is placed on "sufficiently" suppressing freckles since not a single melt condition could be identified that eliminated these defects entirely. Hence, it appears that it is not so much the presence or absence of freckles, but their extent and dispersion that control further process capability of the ESR ingot.
Another noteworthy finding of this study concerns the extent of macro-segregation of niobium encountered at certain re-melting conditions (see Table 1). This pronounced center segregation of $\mathrm{Nb}$ in ESR alloy 718 may be discussed in the context of melt pool dynamics.

The shallow, bowl-shaped pools generated by relatively low melt rates were found to be associated with a significant enrichment of the ingot center with niobium, and predominantly vertical freckle channels. Clearly, as indicated by the straight lines in Fig. 5., no true steadystate melt pool was attained after re-melting several ingot diameters worth of metal. It is speculated that a fairly quiescent pool existed under these conditions, which was conducive to gravity-driven settlement of the higherdensity (Nb-rich) interdendritic liquid at the bottom of the pool. 
At relatively high melt rates, a highly turbulent pool may have existed that resulted in good stirring - hence the insignificant compositional differences between center and edge. The qualitatively different pool shape and orientation of the freckle channels seem to support this conjecture. Presently, it is not known what might have triggered this switch in modes.

\section{Summary}

1. The effect of melt conditions, chiefly melt rate, on the structural integrity of nominally (33-35) inch (838-890 $\mathrm{mm}$ ) diameter ESR alloy 718 ingot was investigated.

2. At relatively low melt rates, the melt pools were shallow and bowl-shaped, and associated with predominantly vertical freckle channels. A significant enrichment of niobium at the bottom of the pool, which increased as the pool gradually deepened, was measured under these conditions.

3. At the highest melt rate, the melt pool was found to be quite deep and V-shaped as expected, but was not accompanied by an enrichment in niobium. At the same time, the freckle channels reoriented themselves, following the pool contour.

4. None of the melt rates studied resulted in macro-segregation-free, i.e., freckle-free, ingot. Even excessive high-temperature anneals were found to be ineffective in solutioning these defects.
5. Nevertheless, melt conditions were identified under which the ingot exhibited sufficient ductility and could, thus, be processed further into sound triple-melted 30 inch $(760 \mathrm{~mm})$ diameter VIM + ESR + VAR product.

\section{Acknowledgments}

The authors acknowledge helpful discussions with J. J. deBarbadillo and W. Yang.

\section{References}

[1] R. C. Schwant et al., Proc. Intl. Symp. on Superalloys 718, 625, 706 and Various Derivatives, ed. E. Loria, TMS / Warrendale, 1997, p. 141.

[2] S.T. Wlodek and R.D. Field, Proc. International Symposium on Superalloys 718, 625, 706 and Various Derivatives, TMS / Warrendale, 1994, p. 167.

[3] B. Sundman, B. Jansson, and J.O. Anderson, CALPHAD 9 (1985) 153.

[4] M.D. Evans and G.E. Kruzynski, Proc. Sixth International Symposium on Superalloys, TMS / Warrendale, 1988, p. 91. 
